\title{
Accurate Translucent Material Rendering under Spherical Gaussian Lights
}

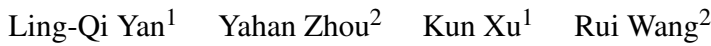 \\ ${ }^{1}$ TNList, Department of Computer Science and Technology, Tsinghua University, Beijing \\ ${ }^{2}$ Department of Computer Science, University of Massachusetts, Amherst
}

\begin{abstract}
In this paper we present a new algorithm for accurate rendering of translucent materials under Spherical Gaussian (SG) lights. Our algorithm builds upon the quantized-diffusion BSSRDF model recently introduced in [dI11]. Our main contribution is an efficient algorithm for computing the integral of the BSSRDF with an SG light. We incorporate both single and multiple scattering components. Our model improves upon previous work by accounting for the incident angle of each individual SG light. This leads to more accurate rendering results, notably elliptical profiles from oblique illumination. In contrast, most existing models only consider the total irradiance received from all lights, hence can only generate circular profiles. Experimental results show that our method is suitable for rendering of translucent materials under finite-area lights or environment lights that can be approximated by a small number of SGs.
\end{abstract}

Categories and Subject Descriptors (according to ACM CCS): I.3.7 [Computer Graphics]: Three-Dimensional Graphics and Realism—Color, shading, shadowing, and texture

\section{Introduction}

Realistic image synthesis often requires accurate simulation of translucent materials. At the appropriate scale level, many materials exhibit translucency effects, giving them a smooth and soft look, blurring small geometric details. These effects account for the natural appearance of media such marble, jade, wax, leaves, milk, fruits, and human skin. To simulate translucency, a common approach is to model the transfer of light through the material interior (subsurface level) using a BSSRDF (bidirectional scattering-surface reflectance distribution function). Generally, the BSSRDF describes the linear relationship of the incident light at a surface point to the outgoing light at any other point on the surface.

An accurate and efficient BSSRDF model has been a central area of research for the past two decades. By assuming homogeneous media, Jensen et al. [JMLH01] introduced the first practical formula of BSSRDF as the sum of a single scattering component and a multiple scattering component based on a diffuse dipole approximation. The dipole approximation is fast to compute but is derived from a semi-infinite media, which compromises accuracy when the geometry is complex. This model was then extended to multi-layer materials using a multipole approximation [DJ05]. Later the dipole, multipole, and a new quadpole model are combined in a photon diffusion framework in [DJ07] to further improve the accuracy. In addition, Li et al. [LPT05] proposed a hybrid Monte Carlo technique using modified diffusion theory; and Donner et al. [DLR $\left.{ }^{*} 09\right]$ presented an empirical BSSRDF model built upon extensive Monte Carlo simulation.

Most recently, d'Eon et al. [dI11] introduced a quantizeddiffusion BSSRDF model. This new analytic BSSRDF accounts for scattering within multilayer translucent materials with arbitrary levels of absorption, very thin layers, and under high-frequency illumination. They assume a vertical downward incident illumination direction and analytically integrate point sources along the incident path by approximating the diffusion function with quantized Gaussian functions. Our model builds upon their method. The first contribution of our work is to improve the BSSRDF model in [dI11] by accounting for oblique incident lighting directions instead of assuming a vertical only direction. The motivation is to accurately handle oblique illumination, which leads to shifted and asymmetric reflectance profiles [DJ07]. We demonstrate that the integration over an oblique lighting path can also be evaluated analytically. 
The second contribution of our work is an efficient method for quickly computing the integral of the BSSRDF with Spherical Gaussian (SG) lights. This is motivated by the observation that while much existing work can efficiently render translucent materials under simple lighting (e.g. a few point or directional lights), it remains a challenge to simulate translucent appearance under complex environment lighting. It is well-known that environment lighting is important for capturing the appearance of materials in natural illumination conditions; at the same time, it is also very expensive to simulate due to the large area of the lights. An effective solution is to approximate the environment lighting as a set of SGs, yielding a low-dimensional representation. Combined with the SG representation of the BSSRDF, this approach enables efficient run-time evaluation of the integral of complex lighting with BSSRDF.

To summarize, this paper presents a new algorithm for accurate rendering of translucent materials under Spherical Gaussian lights. Our specific contributions are: 1) we extend the BSSRDF introduced in [dI11] to allow efficient evaluation of its integral with SG lights at varying incident angles; 2) our model includes both single and multiple scattering, and experimental results show that it can accurately render translucent materials under finite-area lights and environment lights approximated by a small number of SGs.

\section{Related Works}

The reflectance of light due to subsurface scattering is often described by the BSSRDF, which characterizes how the incident light at a surface point of the translucent material influences the outgoing light at other surface points. Given a BSSRDF model, it is possible to use Monte Carlo simulation to accurately render translucency effects [HK93, DEL*99, PH00, LPT05]. However, this incurs high computation cost and the resulting images are prone to noise.

Homogeneous Materials. For efficiency reasons, subsurfaces scattering effects are often divided into two components: single scattering and multiple scattering. Single scattering accounts for light that has been scattered exactly once inside the media, and is usually fast to compute. Multiple scattering accounts for light that has gone through a large number of scattering events, which makes it possible to use simplifying assumptions to speed up the computation. Stam [Sta95] approximates multiple scattering effects by a diffusion process. Taking advantage of the diffusion process and assuming a semi-infinite homogeneous media, Jensen et al. [JMLH01] presented a dipole approximation model, which speeds up the computation of multiple scattering by orders of magnitude compared to Monte Carlo methods. The dipole model converts the irradiance at a surface point into two point sources, one above and one below the surface. The contribution from each point source is then evaluated as the gradient of a diffusion function (or fluence). Since evaluating the outgoing radiance requires integrating the contribu- tions from all incident points, hierarchical summation techniques [JB02] can be adopted to accelerate this computation.

In [DJ05], Donner et al. proposed a multi-pole model for rendering thin and multilayered translucent materials. In [dLE07], d'Eon et al. proposed an efficient multi-layered model by approximating diffusion profiles using sums of Gaussians. Recently, d'Eon et al. [dI11] proposed a quantized diffusion based BSSRDF model. They place an infinite number of point light sources alone the vertical incident light path, which requires computing an itnegral over the path. The integral is evaluated analytically by approximating the diffusion function with quantized Gaussian functions. None of the above methods considers the different incident angle of lights, thus they always produce symmetric (circular) reflectance profiles. In reality, however, subtle asymmetric (elliptical) profiles are usually observed under oblique lighting directions. To address this missing effect, Donner et al. [DJ07] place exponentially attenuated point lights along the refracted light path instead of on the vertical line. While this produces elliptical profiles, it requires an numerical integral over the incident light path, which is expensive and prone to sampling noise. In contrast, we evaluate the integral analytically, thus our method is less prone to numerical sampling artifacts.

Data-driven techniques have also been studied to model the BSSRDF. For example, Donner et al. [DLR*09] proposed an empirical BSSRDF model for semi-indefinite homogeneous materials by fitting Monte Carlo simulated data.

Heterogeneous materials. For realistic rendering of translucency, one must consider spatially-varying material properties. This is known as heterogeneous materials, which have been the focus of much recent work. Tong et al. [TWL*05] combined the dipole model with bidirectional texture functions to simulate quasi-homogeneous materials. Peers et al. $\left[\mathrm{PvBM}^{*} 06\right]$ proposed a compact representation for heterogeneous materials using matrix factorization. Wang et al. [WZT*08] proposed a method for modeling and rendering heterogeneous materials based on a volumetric representation and the diffusion equation. Song et al. [STPP09] introduced a novel representation for editing measured heterogeneous materials by decomposing a global BSSRDF into products of local functions. Wang et al. [WWH*10] presented a robust method for rendering heterogenous translucent objects with complex shapes and topology. Jakob et al. [JAM*10] proposed an anisotropic dipole model for rendering anisotropic translucent materials. Li et al. [LSR*12] presented an interactive method for rendering translucent cutouts. Moreover, many recent methods $\left[\mathrm{DWd}^{*} 08\right.$, $\mathrm{GHP}^{*} 08, \mathrm{JSB}^{*} 10$ ] have been introduced to model and render human skins or faces, which are typically modeled as heterogeneous translucent materials.

Translucent rendering under environment lights. Environment lighting is important for capturing the realistic appearance of materials under natural illumination conditions. 


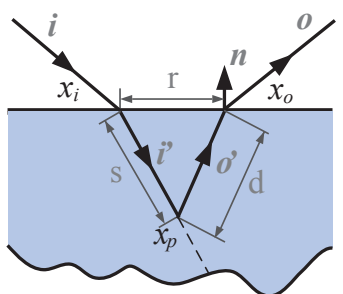

(a)

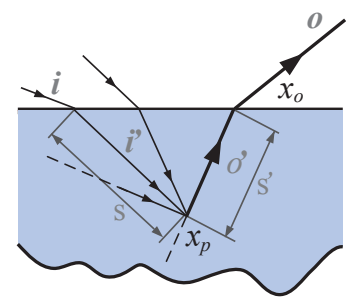

(b)
Figure 1: Illustration of scattering paths for (a) multiple scattering and (b) single scattering.

While a number of previous papers have studied realistic translucent material rendering and editing under environment lighting [WTL05, $\mathrm{XGL}^{*} 07, \mathrm{WCPW}^{*} 08$ ], these methods are all based on precomputed radiance transfer, which requires a large amount of precomputed data and are not suitable for dynamic scenes.

Spherical Gaussian (SG), also known as spherical radial basis function, is commonly used to represent spherical functions such as environment maps [TS06, XMR*11, RZL $\left.{ }^{*} 10\right]$ and BRDFs [GKD07, $\mathrm{WRG}^{*} 09$ ]. It often provides more flexibility and accuracy than other spherical basis functions, such as spherical harmonics, wavelets, and spherical piecewise constants [XJF*08]. Due to its variable support size, SG is particularly suitable for approximating functions at all-frequency scales. Moreover, SG has closed-form solutions for function multiplications and product integrals, which make it a suitable choice for rendering applications.

\section{Backgrounds}

In this section, we review the necessary background knowledge for modeling and approximating BSSRDFs. The list of symbols and notations are summarized in Table 1. Assuming an incident SG light at point $x_{i}$, we compute the outgoing radiance at an exit point $x_{o}$ in outgoing direction $\mathbf{o}$ as the sum of a multiple scattering component $L^{D}$ and a single scattering component $L^{(1)}$ :

$$
L_{o}\left(x_{o}, \mathbf{o}\right)=L^{D}\left(x_{o}, \mathbf{o}\right)+L^{(1)}\left(x_{o}, \mathbf{o}\right)
$$

Multiple Scattering. Under an SG light, the multiple scattering component is defined as [dI11]:

$$
L^{D}\left(x_{o}, \mathbf{o}\right)=\int_{\Omega} G\left(\mathbf{i} ; \mathbf{i}_{j}, \lambda_{j}\right)\left(F \int_{0}^{\infty} Q(s) R(d) \mathrm{d} s\right) \mathrm{d} \mathbf{i}
$$

As shown in Figure 1(a), $\mathbf{i}$ is an incident direction, $G\left(\mathbf{i} ; \mathbf{i}_{j}, \lambda_{j}\right)=\exp \left(\lambda_{j}\left(\mathbf{i} \cdot \mathbf{i}_{j}-1\right)\right)$ is the incident SG light where $\mathbf{i}_{j}$ is the center direction and $\lambda_{j}$ is the bandwidth; $F=F_{t}\left(x_{i}, \mathbf{i}\right) F_{t}\left(x_{o}, \mathbf{0}\right)$ is the product of two Fresnel transmittance terms; $s$ is the distance from the (virtual) point light source $x_{p}$ to the incident point $x_{i} ; d$ is the distance from $x_{p}$ to the exit point $x_{o}$; in addition, $Q(s)$ is the extended point

\begin{tabular}{ll}
\hline$F_{t}, F_{r}$ & Fresnel transmittance and reflectance \\
$\eta$ & Index of refraction \\
$\sigma_{s}, \sigma_{a}$ & Scattering and absorption coefficients \\
$g$ & Mean cosine of scattering angle \\
$C_{\phi}, C_{E}$ & Constants only dependent on $\eta$ \\
$x_{i}, x_{o}, \mathbf{i}, \mathbf{o}$ & Incident/outgoing points and directions \\
$\mathbf{i}^{\prime}, \mathbf{o}^{\prime}$ & Refracted incident/outgoing directions \\
$\mathbf{i}_{2}$ & Direction from $x_{i}$ to $x_{o}$ \\
$\mathbf{i}_{\mathbf{j}}, \lambda_{j}$ & Center and bandwidth of an incident SG light \\
$\mathbf{i}_{\mathbf{j}}^{\prime}, \lambda_{j}^{\prime}$ & Center and bandwidth of a refracted SG light \\
\hline$\sigma_{t}=\sigma_{a}+\sigma_{s}, \sigma_{s}^{\prime}=(1-g) \sigma_{s}, \sigma_{t}^{\prime}=\sigma_{a}+\sigma_{s}^{\prime}$ \\
$F=F_{t}\left(x_{i}, \mathbf{i}\right) F_{t}\left(x_{o}, \mathbf{o}\right)$ \\
$D=\left(2 \sigma_{a}+\sigma_{s}^{\prime}\right) /\left(3\left(\sigma_{a}+\sigma_{s}^{\prime}\right)^{2}\right)$ \\
$G\left(\mathbf{i} ; \mathbf{i}_{1}, \lambda\right)=\exp \left[\lambda\left(\mathbf{i} \cdot \mathbf{i}_{1}-1\right)\right] \quad$ SG definition \\
$g(x ; u, \lambda)=\exp \left[-\lambda(x-u)^{2}\right] \quad$ 1D Gaussian definition \\
\hline
\end{tabular}

Table 1: List of symbols and notations.

source function which describes the intensity of the point light source $x_{p}$; and $R(d)$ is the exitance function which describes the exitance value at point $x_{o}$ from $x_{p}$. The $Q(s)$ and $R(d)$ functions will be explained in more details below.

Note that the inner integral in Equation 2 integrates along the refracted incident path, and the outer integral integrates over the incident direction $\mathbf{i}$ on the upper hemisphere. For simplicity, we rewrite the integral in terms of refracted incident direction $\mathbf{i}^{\prime}$ instead of incident direction $\mathbf{i}$, and approximate refracted incident radiance inside the medium again using an SG (see detailed derivations in Appendix E):

$$
L^{D}\left(x_{o}, \mathbf{o}\right)=\int_{\Omega} \int_{0}^{\infty} F G\left(\mathbf{i}^{\prime} ; \mathbf{i}_{\mathbf{j}}^{\prime}, \lambda_{j}^{\prime}\right) Q(s) R(d) \mathrm{d} s \mathrm{~d} \mathbf{i}^{\prime}
$$

where $\mathbf{i}_{j}^{\prime}, \lambda_{j}^{\prime}$ are the center and bandwidth of the approximate refracted SG light respectively. Strictly speaking, the refracted light caused by an incident SG light is not necessarily an SG, but we find this approximation to work well in practice, especially if the support size of the incident SG is small.

Extended Point Source Function $Q(s)$. In previous work, the dipole model [JMLH01] or multi-pole model [DJ05] has been commonly adopted for translucent material rendering. However, these models typically only consider the total irradiance received at an incident point, ignoring the incident angle of each individual light. As a result, they lead to incorrect renderings for oblique illumination. To consider oblique illumination, we follow [DJ07] to define an extended source function $Q(s)$ along the refracted incident path:

$$
Q(s)=Q e^{-\sigma_{t}^{\prime} s}
$$

where $Q$ is the irradiance at incident point $x_{i}$, and $\sigma_{t}^{\prime}$ is the reduced extinction coefficient (see Table 1).

Exitance Function $R(d)$. Recall that the exitance function 
$R(d)$ describes the contribution of a point source $x_{p}$ underneath the surface to an exit point $x_{o}$ on the surface. Following [dI11], we define $R(d)$ as a linear combination of fluence and flux:

$$
R(d)=C_{\phi} \phi(d)+C_{E}(-D(\mathbf{n} \cdot \nabla \phi)(d))
$$

where $d$ is the distance from $x_{p}$ to $x_{o}$; the $C_{\phi}, C_{E}$ terms are two constants which are only dependent on the refractive index [dI11]; $\mathbf{n}$ is the surface normal at $x_{o}$; and $\phi(d)$ is the diffusion function. Specifically, the diffusion function describes the fluence of an isotropic point light source in an infinite medium, and is usually computed as:

$$
\phi(d)=\frac{1}{4 \pi D} \cdot \frac{e^{-\sqrt{\frac{\sigma_{a}}{D}} d}}{d}
$$

where $D$ is the diffusion constant, and $\sigma_{a}$ is the absorption coefficient. This function can be accurately approximated by a sum of Gaussians [dLE07]:

$$
\phi(d) \approx \sum_{k} a_{k} g\left(d ; 0, \lambda_{k}\right)
$$

where $g\left(d ; 0, \lambda_{k}\right)=\exp \left(-\lambda_{k}(d-0)^{2}\right)$ is a 1D Gaussian with bandwidth $\lambda_{k}$, and $a_{k}$ is the corresponding coefficient. These parameters (i.e. bandwidth and coefficients) can be obtained by using the quantized diffusion method [dI11] or by offline fitting [dLE07]. In our implementation, we follow [dLE07] to obtain these parameters by function fitting. To do so we require a precomputation stage to optimally fit these parameters. Empirically we found that a small number of Gaussian (e.g. $4 \sim 8$ ) terms suffice. While this number is smaller than reported in [dI11], our results (in terms of both function plots and rendering quality) match the ground truth very well.

Single Scattering. The single scattering component $L^{(1)}\left(x_{o}, \mathbf{o}\right)$ in Equation 2 is defined as [JMLH01]:

$$
L^{(1)}\left(x_{o}, \mathbf{o}\right)=\sigma_{s} \int_{0}^{\infty} F_{t} E\left(s^{\prime}\right) \int_{\Omega} p G\left(\mathbf{i}^{\prime} ; \mathbf{i}_{\mathbf{j}}^{\prime}, \lambda_{j}^{\prime}\right) E(s) V\left(\mathbf{i}^{\prime}\right) \mathrm{d} \mathbf{i}^{\prime} \mathrm{d} s^{\prime}
$$

where $\sigma_{s}$ is the scattering coefficient, and $F_{t}=F_{t}\left(x_{o}, \mathbf{o}\right)$ is the Fresnel transmittance term. As shown in Figure 1(b), $s^{\prime}$ is the distance from the point source $x_{p}$ to the exit point $x_{o}$, and $E\left(s^{\prime}\right)=\exp \left(-\sigma_{t} s^{\prime}\right)$ is the attenuation factor.

In the inner integral, $p=p\left(\mathbf{i}^{\prime}, \mathbf{o}^{\prime}\right)$ is the phase function, $G\left(\mathbf{i}^{\prime} ; \mathbf{i}_{\mathbf{j}}^{\prime}, \lambda_{j}^{\prime}\right)$ is the refracted incident SG light, $s$ is the distance from $x_{p}$ to the surface along the refracted incident direction $\mathbf{i}^{\prime}$, and $V\left(\mathbf{i}^{\prime}\right)$ is the binary visibility function towards $\mathbf{i}^{\prime}$, which accounts for the occlusion of the incident light before reaching the surface. Note that in contrast to multiple scattering, here the outer integral integrates along the refracted outgoing path $\mathbf{o}^{\prime}$.

Summary. Assuming incident light is an SG light $G\left(\mathbf{i} ; \mathbf{i}_{j}, \lambda_{j}\right)$, we approximate the (warped) refracted incident light also by an SG function $G\left(\mathbf{i}^{\prime} ; \mathbf{i}_{\mathbf{j}}^{\prime}, \lambda_{j}^{\prime}\right)$. In the following sections, we describe in details how to approximate the multiple scattering integral (Equation 3) in Section 4, and the single scattering integral (Equation 8) in Section 5. We show that after several validated mathematical approximations, both integrals can be efficiently evaluated, resulting in a practical algorithm for rendering BSSRDF under SG lights.

\section{Approximating Multiple Scattering}

In this section, we explain how to approximate the multiple scattering integral in Equation 3. Since the exitance function $R(d)$ is a linear combination of the diffusion function $\phi$ and its gradient( Equation 5), we can substitute Equation 5 into Equation 3. Furthermore, since the Fresnel term $F$ is rather smooth compared to spherical Gaussian or exponential functions, we can safely pull it out of the integral:

$$
L^{D}\left(x_{o}, \mathbf{o}\right) \approx F C_{\phi} L_{\phi}+F D C_{E} L_{E}
$$

where the fluence integral $L_{\phi}$ and the flux integral $L_{E}$ are defined as:

$$
\begin{aligned}
& L_{\phi}=\int_{\Omega} \int_{0}^{\infty} G\left(\mathbf{i}^{\prime} ; \mathbf{i}_{\mathbf{j}}^{\prime}, \lambda_{j}^{\prime}\right) Q(s) \phi(d) \mathrm{d} s \mathrm{di} \mathbf{i}^{\prime} \\
& L_{E}=\int_{\Omega} \int_{0}^{\infty} G\left(\mathbf{i}^{\prime} ; \mathbf{i}_{\mathbf{j}}^{\prime}, \lambda_{j}^{\prime}\right) Q(s)(-\mathbf{n} \cdot \nabla \phi)(d) \mathrm{d} s \mathrm{di}^{\prime}
\end{aligned}
$$

where $\mathbf{n}$ is the surface normal. Below we describe how to evaluate the fluence integral $L_{\phi}$ and the flux integral $L_{E}$ respectively.

\subsection{Approximating the Fluence Integral}

From Figure 1(a), we see that the distance $d$ from $x_{p}$ to $x_{o}$ can be written as (using the law of cosine):

$$
d=\sqrt{s^{2}+r^{2}-2 s r\left(\mathbf{i}^{\prime} \cdot \mathbf{i}_{2}\right)}
$$

where $r$ is the distance from $x_{i}$ to $x_{o}$, and $\mathbf{i}_{\mathbf{2}}$ is the unit vector from $x_{i}$ to $x_{o}$. Given the approximation of the diffusion function by sum of Gaussians $\phi(d) \approx \sum_{k} a_{k} g\left(d ; 0, \lambda_{k}\right)$ (Equation 7), we can express the fluence integral $L_{\phi}$ in Equation 10 as (for simplicity we omit the summation $\sum_{k}(\cdot)$ and the coefficients $a_{k}$ over index $k$ below):

$$
\begin{aligned}
& L_{\phi} \approx \int_{\Omega} \int_{0}^{\infty} G\left(\mathbf{i}^{\prime} ; \mathbf{i}_{\mathbf{j}}^{\prime}, \lambda_{j}^{\prime}\right) Q(s) g\left(d ; 0, \lambda_{k}\right) \mathrm{d} s \mathrm{~d} \mathbf{i}^{\prime} \\
= & \int_{\Omega} \int_{0}^{\infty} G\left(\mathbf{i}^{\prime} ; \mathbf{i}_{\mathbf{j}}^{\prime}, \lambda_{j}^{\prime}\right) Q(s) e^{\lambda_{k}\left(-\left(s^{2}+r^{2}-2 s r\left(\mathbf{i}^{\prime} \cdot \mathbf{i}_{2}\right)\right)\right)} \mathrm{d} s \mathrm{~d} \mathbf{i}^{\prime} \\
= & \int_{0}^{\infty} Q(s) e^{-\lambda_{k}\left(s^{2}+r^{2}-2 s r\right)}\left(\int_{\Omega} G\left(\mathbf{i}^{\prime} ; \mathbf{i}_{\mathbf{j}}^{\prime}, \lambda_{j}^{\prime}\right) e^{2 s r \lambda_{k}\left(\mathbf{i}^{\prime} \cdot \mathbf{i}_{2}-1\right)} \mathrm{d} \mathbf{i}^{\prime}\right) \mathrm{d} s \\
= & \int_{0}^{\infty} Q(s) e^{-\lambda_{k}\left(s^{2}+r^{2}-2 s r\right)} \mathbf{N} \mathrm{d} s
\end{aligned}
$$

where $\mathbf{N}$ is the inner integral over $\mathbf{i}^{\prime}$, computed as:

$$
\begin{aligned}
\mathbf{N} & =\int_{\Omega} G\left(\mathbf{i}^{\prime} ; \mathbf{i}_{j}^{\prime}, \lambda_{j}^{\prime}\right) e^{2 s r \lambda_{k}\left(\mathbf{i}^{\prime} \cdot \mathbf{i}_{2}-1\right)} \mathrm{di}^{\prime} \\
& =\int_{\Omega} G\left(\mathbf{i}^{\prime} ; \mathbf{i}_{\mathbf{j}}^{\prime}, \lambda_{j}^{\prime}\right) G\left(\mathbf{i}^{\prime} ; \mathbf{i}_{2}, \lambda_{k}^{\prime}\right) \mathrm{d} \mathbf{i}^{\prime}
\end{aligned}
$$

where the effective bandwidth $\lambda_{k}^{\prime}=2 s r \lambda_{k}$. This equation is integrated over the refracted incident direction $\mathbf{i}^{\prime}$ on the 
lower hemisphere. In general, since the support of an SG is typically small compared to the size of a hemisphere, the value of the SG on the other hemisphere is usually very close to zero. Therefore, we can express the integral over the entire sphere (instead of only the lower hemisphere). The reason to do so is to take advantage of the properties of SG to simplify the computations later. Specifically, it is well known that the product of two SGs is still an SG, and the integral of an SG over the whole sphere has analytic expression (refer to Appendix A, B, C). Therefore, given these properties, we have:

$$
\mathbf{N}=2 \pi \frac{e^{\lambda_{3}-\lambda_{j}^{\prime}-\lambda_{k}^{\prime}}}{\lambda_{3}}\left(1-e^{-2 \lambda_{3}}\right)
$$

where $\lambda_{3}=\sqrt{\lambda_{j}^{\prime 2}+\lambda_{k}^{\prime 2}+2 \lambda_{j}^{\prime} \lambda_{k}^{\prime}\left(\mathbf{i}_{\mathbf{j}}^{\prime} \cdot \mathbf{i}_{2}\right)}$. Note that $\mathbf{N}$ depends on three parameters: the bandwidth of the SG light $\lambda_{j}^{\prime}$, the effective bandwidth $\lambda_{k}^{\prime}$, and the dot product $\left(\mathbf{i}_{\mathbf{j}}^{\prime} \cdot \mathbf{i}_{\mathbf{2}}\right)$. Through experimental results, we find that $\mathbf{N}$ falls off exponentially when $\lambda_{k}^{\prime}$ increases, so we can naturally approximate $\mathbf{N}$ as a sum of exponential functions of $\lambda_{k}^{\prime}$ :

$$
\mathbf{N} \approx \sum_{m} p_{m}^{\phi}\left(\lambda_{j}^{\prime}, \mathbf{i}_{\mathbf{j}}^{\prime} \cdot \mathbf{i}_{\mathbf{2}}\right) \exp \left(-q_{m}^{\phi}\left(\lambda_{j}^{\prime}, \mathbf{i}_{\mathbf{j}}^{\prime} \cdot \mathbf{i}_{\mathbf{2}}\right) \lambda_{k}^{\prime}\right)
$$

where $p_{m}^{\phi}, q_{m}^{\phi}$ are parameters to be fitted during precomputation step, and they are stored as a $2 \mathrm{D}$ table of $\lambda_{j}^{\prime}$ and $\left(\mathbf{i}_{\mathbf{j}}^{\prime} \cdot \mathbf{i}_{\mathbf{2}}\right)$. In Figure 2 we validate this approximation. As shown in the figure, usually 4 terms are sufficiently accurate to capture $\mathbf{N}$ over a large range.

Now substitute Equation 12 into Equation 11, and replace the effective bandwidth $\lambda_{k}^{\prime}$ by $2 s r \lambda_{k}$ (by definition), we have (for simplicity the summation $\sum_{m}(\cdot)$ and the coefficients $p_{m}^{\phi}$ of index $m$ are omitted below):

$$
\begin{aligned}
L_{\phi} & \approx \int_{0}^{\infty} e^{-\sigma_{t}^{\prime} s} e^{-\lambda_{k}\left(s^{2}+r^{2}-2 s r\right)} e^{-2 q_{m}^{\phi} r \lambda_{k} s} \mathrm{~d} s \\
& =\int_{0}^{\infty} e^{-\left(\lambda_{k} s^{2}+\left(2 \lambda_{k}\left(q_{m}^{\phi}-1\right) r+\sigma_{t}^{\prime}\right) s+\lambda_{k} r^{2}\right)} \mathrm{d} s
\end{aligned}
$$

Since the integrand is a 1D Gaussian of $s$, this half-infinite integral has an analytic solution as shown in Appendix D.

\subsection{Approximating the Flux Integral}

Now we explain how to evaluate the flux integral in Equation 9 . To begin, we note that the directional derivative of a $1 \mathrm{D}$ Gaussian is evaluated as:

$$
\mathbf{n} \cdot \nabla g\left(d ; 0, \lambda_{k}\right)=2 \lambda_{k} s\left(\mathbf{i}^{\prime} \cdot \mathbf{n}\right) g\left(d ; 0, \lambda_{k}\right)
$$

Given that the diffusion function is approximated as a sum of Gaussians $\phi(d) \approx \sum_{k} a_{k} g\left(d ; 0, \lambda_{k}\right)$ (Equation 7), the directional derivative of the diffusion function $(-\mathbf{n} \cdot \nabla \phi)(d)$ in Equation 9 can now be written as:

$$
(-\mathbf{n} \cdot \nabla \phi)(d) \approx \sum_{k} 2 a_{k} \lambda_{k} s\left(\mathbf{i}^{\prime} \cdot \mathbf{n}\right) g\left(d ; 0, \lambda_{k}\right)
$$

Substituting Equation 14 into Equation 9 yields (again, omitting the summation $\sum_{k}(\cdot)$ over index $k$ and coefficients $a_{k}$ ):

$L_{E} \approx 2 \lambda_{k} \int_{\Omega} \int_{0}^{\infty} G\left(\mathbf{i}^{\prime} ; \mathbf{i}_{\mathbf{j}}^{\prime}, \lambda_{j}^{\prime}\right) Q(s)\left(\mathbf{i}^{\prime} \cdot \mathbf{n}\right) e^{\lambda_{k}\left(-\left(s^{2}+r^{2}-2 s r\left(\mathbf{i}^{\prime} \cdot \mathbf{i}_{2}\right)\right)\right)} \mathrm{d} s \mathrm{di} \mathbf{i}^{\prime}$

Similar to how we handled the fluence integral $L_{\phi}$ in Equation 11, the flux integral $L_{E}$ can be re-written as (by changing the order of the integrals):

$$
L_{E} \approx 2 \lambda_{k} \int_{0}^{\infty} Q(s) e^{-\lambda_{k}\left(s^{2}+r^{2}-2 s r\right)} \mathbf{M} \mathrm{d} s
$$

where $\mathbf{M}$ is the inner integral over $\mathbf{i}^{\prime}$ defined as:

$$
\mathbf{M}=\int_{\Omega} G\left(\mathbf{i}^{\prime} ; \mathbf{i}_{\mathbf{j}}^{\prime}, \lambda_{j}^{\prime}\right) G\left(\mathbf{i}^{\prime} ; \mathbf{i}_{2}, \lambda_{k}^{\prime}\right)\left(\mathbf{i}^{\prime} \cdot \mathbf{n}\right) \mathrm{d} \mathbf{i}^{\prime}
$$

where the effective bandwidth $\lambda_{k}^{\prime}=2 s r \lambda_{k}$. Different from the fluence inner integral $\mathbf{N}$ in Equation 12, the flux inner integral $\mathbf{M}$ has an additional cosine factor $\left(\mathbf{i}^{\prime} \cdot \mathbf{n}\right)$. It also has an approximate analytic solution (see detailed derivations in Appendix C):

$$
\mathbf{M} \approx\left(\lambda_{j}^{\prime}\left(\mathbf{i}_{\mathbf{j}}^{\prime} \cdot \mathbf{n}\right)+\lambda_{k}^{\prime}\left(\mathbf{i}_{\mathbf{2}} \cdot \mathbf{n}\right)\right) \mathbf{M}_{\mathbf{i}}
$$

where

$$
\begin{gathered}
\mathbf{M}_{\mathbf{i}}=2 \pi \frac{e^{\lambda_{3}-\lambda_{j}^{\prime}-\lambda_{k}^{\prime}}}{\lambda_{3}^{2}}\left(1-e^{-2 \lambda_{3}}\right), \\
\lambda_{3}=\sqrt{\lambda_{j}^{\prime 2}+\lambda_{k}^{\prime 2}+2 \lambda_{j}^{\prime} \lambda_{k}^{\prime}\left(\mathbf{i}_{\mathbf{j}}^{\prime} \cdot \mathbf{i}_{2}\right)}
\end{gathered}
$$

$\mathbf{M}_{\mathbf{i}}$, being a function that falls off almost exponentially to $\lambda_{k}^{\prime}$, can again be approximated as a sum of exponentials of $\lambda_{k}^{\prime}$ :

$$
\mathbf{M}_{\mathbf{i}} \approx \sum_{m} p_{m}^{E}\left(\lambda_{j}^{\prime}, \mathbf{i}_{\mathbf{j}}^{\prime} \cdot \mathbf{i}_{\mathbf{2}}\right) \exp \left(-q_{m}^{E}\left(\lambda_{j}^{\prime}, \mathbf{i}_{\mathbf{j}}^{\prime} \cdot \mathbf{i}_{\mathbf{2}}\right) \lambda_{k}^{\prime}\right)
$$

where $p_{m}^{E}, q_{m}^{E}$ are fitted parameters estimated in precomputation and stored as a 2D table of $\lambda_{j}^{\prime}$ and $\left(\mathbf{i}_{\mathbf{j}}^{\prime} \cdot \mathbf{i}_{2}\right)$. Similar to before, our experimental results show that using 4 terms is sufficiently accurate for this approximation.

Substitute Equations 17, 18 into Equation 15, and replace $\lambda_{k}^{\prime}$ by $2 s r \lambda_{k}$ (by definition), we have (for simplicity we omit the summation $\sum_{m}(\cdot)$ and the coefficients $p_{m}^{E}$ of index $m$ ):

$$
\begin{aligned}
& L_{E} \approx \lambda_{j}^{\prime}\left(\mathbf{i}_{\mathbf{j}}^{\prime} \cdot \mathbf{n}\right) \int_{0}^{\infty} e^{-\left(\lambda_{k} s^{2}+\left(2 \lambda_{k}\left(q_{m}^{E}-1\right) r+\sigma_{t}^{\prime}\right) s+\lambda_{k} r^{2}\right)} d s \\
& +2 r \lambda_{k}\left(\mathbf{i}_{2} \cdot \mathbf{n}\right) \int_{0}^{\infty} s e^{-\left(\lambda_{k} s^{2}+\left(2 \lambda_{k}\left(q_{m}^{E}-1\right) r+\sigma_{t}^{\prime}\right) s+\lambda_{k} r^{2}\right)} d s
\end{aligned}
$$

The above equation involves a half-infinite integral of a 1D Gaussian as well as the product of a 1D Gaussian with a linear function, both of which have analytic solutions as shown in Appendix D.

Summary. By approximating the diffusion function $\phi(d)$ using sums of 1D Gaussians, and precomputing four 2D tables $p_{m}^{\phi}, q_{m}^{\phi}, p_{m}^{E}, q_{m}^{E}$, the fluence integral $L_{\phi}$ and the flux integral $L_{E}$ can both be approximated analytically, using Equation 13 and Equation 19 respectively. Note that all four 2D tables only need to precomputed once, and remain the same for different scenes and materials. 


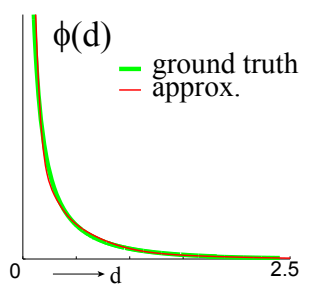

(a)

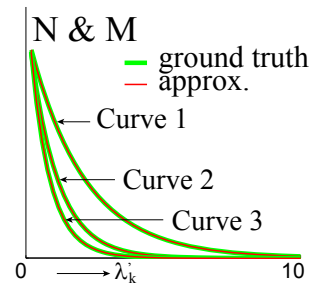

(b)
Figure 2: (a): approximating the diffusion function $\phi$ using sums of Gaussians (Equation 7); (b): approximating the inner integrals $\mathbf{N}$ and $\mathbf{M}$ using sums of exponentials (Equations 12 and 18). Curve 1 plots the fluence inner integral $\mathbf{N}$ with parameters $\lambda_{j}^{\prime}=1000$ and $\left(\mathbf{i}_{\mathbf{j}}^{\prime} \cdot \mathbf{i}_{2}\right)=0.5$; Curve 2 plots the flux inner integral $\mathbf{M}$ with parameters $\lambda_{j}^{\prime}=100$ and $\left(\mathbf{i}_{\mathbf{j}}^{\prime} \cdot \mathbf{i}_{2}\right)=0$; Curve 3 plots the fluence inner integral $\mathbf{N}$ with parameters $\lambda_{j}^{\prime}=10$ and $\left(\mathbf{i}_{\mathbf{j}}^{\prime} \cdot \mathbf{i}_{2}\right)=-0.5$. Note that for all above approximations, the ground truth curves (the green ones) match our approximations (the red ones) very well. We note that the three sets of curves in (b) are normalized in order to display in the same figure.

We would like to note that our method differs from the quantized diffusion model [dI11] in two aspects. First, we consider oblique incident lights, while the quantized model assumes incident lights comes vertically. Second, our method considers SG light, which is suitable for representing environment lights or finite-area lights. From a high level, the quantized model gives an analytic solution for approximating the inner integral over the scattering path, while our proposed method is for approximating the double integral, over both the light direction and the scattering path.

\section{Approximating Single Scattering}

Let us denote the inner integral of the single scattering $L^{(1)}\left(x_{o}, \mathbf{o}\right)$ in Equation 8 using symbol $J$, defined as:

$$
J\left(s^{\prime}\right)=\int_{\Omega} p\left(\mathbf{i}^{\prime}, \mathbf{o}^{\prime}\right) G\left(\mathbf{i}^{\prime} ; \mathbf{i}_{\mathbf{j}}^{\prime}, \lambda_{j}^{\prime}\right) E(s) V\left(\mathbf{i}^{\prime}\right) \mathrm{di}^{\prime}
$$

where $p\left(\mathbf{i}^{\prime}, \mathbf{o}^{\prime}\right)$ is the phase function. $G\left(\mathbf{i}^{\prime} ; \mathbf{i}_{\mathbf{j}}^{\prime}, \lambda_{j}^{\prime}\right)$ is the refracted SG light, $E(s)=\exp \left(-\sigma_{t} s\right)$ is the exponential attenuation term, and $V\left(\mathbf{i}^{\prime}\right)$ is the binary visibility function. By pulling the exponential attenuation term and the visibility term out of the integral, we have:

$$
J\left(s^{\prime}\right) \approx \bar{E} \cdot \bar{V} \int_{\Omega} p\left(\mathbf{i}^{\prime}, \mathbf{o}^{\prime}\right) G\left(\mathbf{i}^{\prime} ; \mathbf{i}_{\mathbf{j}}^{\prime}, \lambda_{j}^{\prime}\right) \mathrm{d} \mathbf{i}^{\prime}
$$

where $\bar{E}$ and $\bar{V}$ are the average attenuation value and average visibility value, respectively. We approximate the average attenuation value directly using the value at the SG center $\bar{E} \approx E\left(s\left(\mathbf{i}_{\mathbf{j}}^{\prime}\right)\right)$. The average visibility $\bar{V}$ value is approximated using a soft shadow technique. In our implementa- tion, we use convolution shadow map [ADM $\left.{ }^{*} 08\right]$ with light radius $\sqrt{1 /\left(2 \lambda_{j}^{\prime}\right)}$ to query the average visibility. We use the Eddington phase function $p\left(\mathbf{i}^{\prime}, \mathbf{o}^{\prime}\right)=\left(1+3 g\left(\mathbf{i}^{\prime} \cdot \mathbf{o}^{\prime}\right)\right) /(4 \pi)$ (where $g$ is the mean cosine of the scattering angle), so $J\left(s^{\prime}\right)$ can be re-written as:

$J\left(s^{\prime}\right) \approx \frac{\bar{E} \cdot \bar{V}}{4 \pi}\left(\int_{\Omega} G\left(\mathbf{i}^{\prime} ; \mathbf{i}_{\mathbf{j}}^{\prime}, \lambda_{j}^{\prime}\right) \mathrm{d} \mathbf{i}^{\prime}+3 g \int_{\Omega}\left(\mathbf{i}^{\prime} \cdot \mathbf{o}^{\prime}\right) G\left(\mathbf{i}^{\prime} ; \mathbf{i}_{\mathbf{j}}^{\prime}, \lambda_{j}^{\prime}\right) \mathrm{d} \mathbf{i}^{\prime}\right)$

Fortunately, both integrals have analytic solutions. Refer to Appendix A for details.

To evaluate the single scattering integral $L^{(1)}\left(x_{o}, \mathbf{0}\right)$, we approximate the outer integral in Equation 8 through an importance sampling approach. We use 32 samples along the outgoing path, with distribution function $e^{-\sigma_{t} s^{\prime}}$. To handle more complicated phase function such as the HenyeyGreenstein phase function, we can approximate it using sum of Gaussians similar to approximating diffusion functions (Equation 7).

\section{Implementation}

Precomputation. Before rendering, we need to first obtain the Gaussian coefficients $a_{k}$ and bandwidths $\lambda_{k}$ for approximating the diffusion function $\phi$ (Equation 7), as well as the 2D tables $p_{m}^{\phi}, p_{m}^{E}, q_{m}^{\phi}, q_{m}^{E}$ of parameters $\lambda_{j}^{\prime}$ and $\left.\mathbf{i}_{\mathbf{j}}^{\prime} \cdot \mathbf{i}_{2}\right)$ $(0 \leq m<4)$ for approximating inner integrals (Equations 12 and 18). All values are obtained through non-linear fitting using Matlab's fminsearch routine. For the four 2D tables, we use a resolution of $256 \times 256$, in which $\lambda_{j}^{\prime}$ is sampled logarithmically from the range $[1,10000]$, and $\mathbf{i}_{\mathbf{j}}^{\prime} \cdot \mathbf{i}_{2}$ is sampled uniformly from the range $[-1,1]$. Note that all tables are pre-computed only once, and can be used for any scene. For environment map lighting, SG lights are obtained through nonlinear fitting following [TS06].

Multiple scattering. For multiple scattering effects, we use Equations 13 and 19 to evaluate the contribution of an incident point $x_{i}$ under SG light to an outgoing point $x_{o}$. Since the derivations are based on a planar geometry assumption, when rendering real 3D models, we use the unit vector from incident point $x_{i}$ to outgoing point $x_{o}$ as surface direction $\mathbf{i}_{2}$. Normals of $x_{i}$ and $x_{o}$ are used for calculating refracted incoming/outgoing light directions, respectively. An average visibility from each SG light is approximated using the convolution shadow map algorithm [ADM*08], in which the support of each SG light is used as area light size to query visibility. An alternative algorithm for handling SG light visibility is to use the visibility distance map [WRG*09], but that would require an additional precomputation step for each scene. Note that contributions from both the positive and negative sources must be taken into account for correct calculation.

Single scattering. For single scattering effects, the inner integral $J\left(s^{\prime}\right)$ is evaluated analytically using Equation 20, 
Ling-Qi Yan, Yahan Zhou, Kun Xu, Rui Wang / Accurate Translucent Material Rendering under Spherical Gaussian Lights
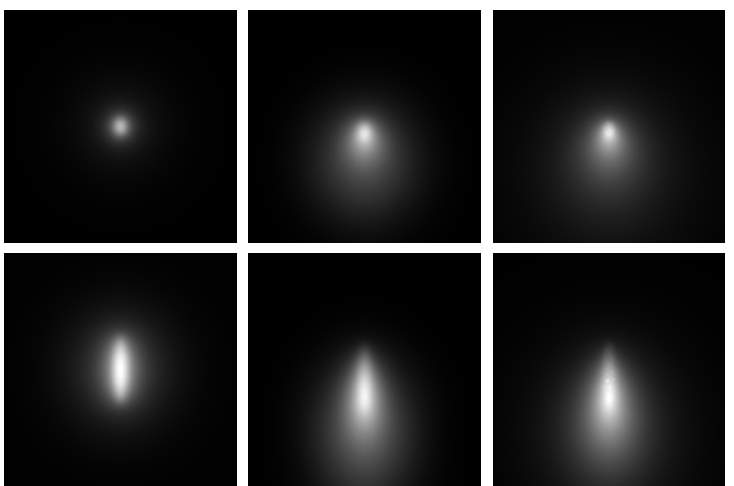

[dI11]

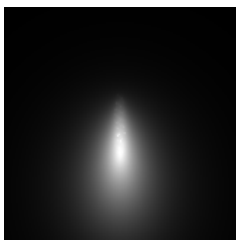

ground truth

Figure 3: Comparison of oblique illumination. The incident angles for the first and second rows are $45^{\circ}$ and $80^{\circ}$, respectively.

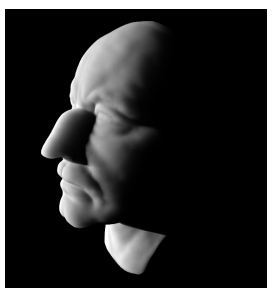

(a)

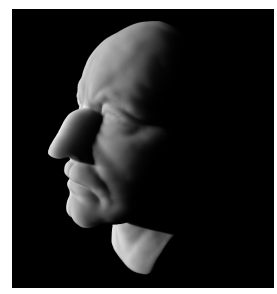

(b)

Figure 4: Comparison of single scattering effects under a single SG light with bandwidth $\lambda_{j}=1000$. (a) our result; (b) ground truth. Note that the difference between our result and the ground truth is almost indistinguishable.

while the final radiance $L^{(1)}\left(x_{o}, \mathbf{o}\right)$ (in Equation 8) is obtained through a numerical integration along the refracted outgoing path. We use 32 samples along the outgoing path, with distribution function $\exp \left(-\sigma_{t} s^{\prime}\right)$. We also incorporate an octree structure [JB02] to accelerate the summation over all incident points. Note that at each octree node, instead of storing an irradiance value, we store the SG light representation, area size, and an average normal direction.

\section{Comparisons and Results}

In this section, we verify the approximations of our method, and compare previous methods with results generated by our method. All results are produced on a PC with Intel Core 2 Duo $3.00 \mathrm{GHz}$ CPU, 6G RAM and an NVIDIA GTX 480 graphics card.

Comparisons. We first mathematically evaluate the accuracy of the approximations used in our method. In Figure 2 (a), we compare our approximated diffusion function using 4 Gaussians to the ground truth; in Figure 2 (b), we compare our approximated inner integrals $\mathbf{N}$ and $\mathbf{M}$ using 4 exponentials (Equations 12 and 16 ) to the ground truth at 3 differ-

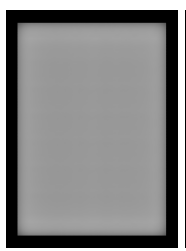

(a)

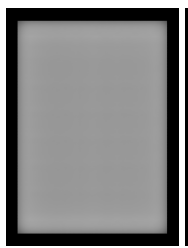

(b)

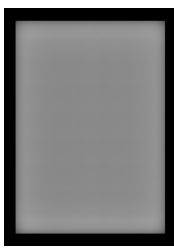

(c)

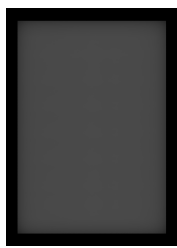

(d)
Figure 5: A thin plate illuminated from behind. (a) ground truth; (b) result by our method; (c) result by the multipole model [DJ05]; (d) result by the dipole model. Note that the difference between our result and the ground truth is subtle.

\begin{tabular}{lccc}
\hline Scene & \#Samples & \#SGs & Render Time \\
\hline Tweety & $139 \mathrm{k}$ & 10 & $8.5 \mathrm{~min}$ \\
Bunny & $35 \mathrm{k}$ & 10 & $5 \mathrm{~min}$ \\
Dragon & $159 \mathrm{k}$ & 20 & $23 \mathrm{~min}$ \\
Statue & $242 \mathrm{k}$ & 1 & $3.5 \mathrm{~min}$ \\
Kitten & $137 \mathrm{k}$ & 10 & $11 \mathrm{~min}$ \\
\hline
\end{tabular}

Table 2: Performances.

ent parameters. Note that in all comparisons, our approximations match the ground truth very well. In our experiments, all results are generated by approximating the diffusion function as 4 terms of Gaussians and by approximating the inner integrals as 4 terms of exponentials.

In Figure 3, we compare oblique illumination effects of our method to [dI11]. An SG light with bandwidth $\lambda=1000$ is used to illuminate a semi-indefinite translucent plane. The incident angles are $45^{\circ}$ and $80^{\circ}$ for the first and second rows, respectively. An oblique incident angle will result in a shifted and asymmetric reflectance lobe. As shown in the figure, our method accurately captures this phenomenon, while [dI11] does not since it ignores the incident angle.

In Figure 4, we compare the rendering results with only single scattering component under a single SG light. Figure 4 (a) shows our result while Figure 4 (b) shows the corresponding ground truth. Our result is visually almost the same with the ground truth.

Results. In Figure 6, we show examples of rendering results using our method under various lightings conditions represented by SG lights, including both multiple and single scattering effects. The performance is reported in Table 2. Note that instead of using only irradiance values at incoming sample points for computing multiple scattering, we use radiance values approximated by SGs, hence supporting oblique incoming direction. This leads to a more accurate approximation as demonstrated in Figure 3.

Extension to a Multi-pole model. Besides handling homogeneous translucent materials, our method can also incorporate the multipole model to handle thin materials or multi- 

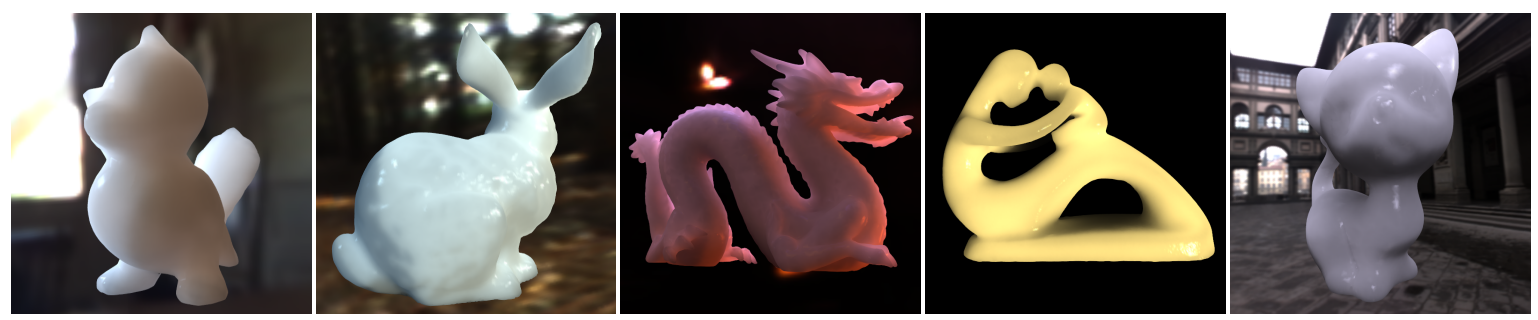

Figure 6: Various rendering results using our method under SG lights.

layers materials similar to [dI11]. The only change is to replace the integration range in Equations 13 and 19 from $[0,+\infty]$ to a finite range, which is determined by the thickness of the material. In Figure 5 (b), we render a thin plate illuminated from behind using our method. To compare, we also show the ground truth image in Figure 5 (a), the rendered results generated using the multipole model [DJ05] in Figure 5 (c) and using the dipole model in Figure 5 (d), respectively. Note that our result and that of [DJ05] both match the ground truth very well, while the result of the dipole model [JMLH01] appears darker.

\section{Conclusion and Future Work}

To summarize, we have presented a new method for accurate rendering of translucent materials under SG light, including both multiple and single scattering components. Compared to existing models [JMLH01, dI11] which only consider the total irradiance received from all incident lights, our method accounts for oblique lighting angles, so it is more accurate and can generate elliptical reflectance profiles. Our main contribution is an efficient method for evaluating the integral of BSSRDF with SG lights. This makes our method suitable for rendering translucent materials under finite-area lights or environment lights that can be approximated by a small number of SGs. Besides, our method can also easily integrate the multipole model to handle thin or multi-layer translucent materials.

Our method is currently limited to homogeneous or multilayer materials. In the future, we would like to investigate ways to extend our method to more complex, heterogeneous translucent materials [ $\left.\mathrm{PvBM}^{*} 06\right]$ or participating media [SZLG10, BGP*10]. Another limitation is that approximating the refracted incident lighting as an SG (as explained in Appendix E) may become inaccurate when the bandwidth parameter is very small (i.e. the SG light is very wide). This can be addressed by constraining the bandwidth of an SG light when fitting the environment map.

Acknowledgements. We thank all the reviewers for their valuable comments and insightful suggestions. This work was supported by the National Basic Research Project of China (Project Number 2011CB302205), the Natural Science Foundation of China (Project Number 61170153), the
National High Technology Research and Development Program of China (Project Number 2012AA011503), and the National Science Foundation of the United States (CCF0746577).

\section{References}

[ADM*08] Annen T., Dong Z., Mertens T., Bekaert P., SEIDEL H.-P., KAUTZ J.: Real-time all-frequency shadows in dynamic scenes. ACM Transactions on Graphics 27, 3 (2008), 34:1-34:8.

[BGP*10] Bernabei D., Ganovelli F., Pietroni N., Cignoni P., PATtanaik S. N., SCOPIGNo R.: Real-time single scattering inside inhomogeneous materials. The Visual Computer 26, 6-8 (2010), 583-593.

[DEL*99] Dorsey J., Edelman A., Legakis J., Jensen H. W., Pedersen H. K.: Modeling and rendering of weathered stone. In Proceedings of SIGGRAPH (1999), pp. 225-234.

[dI11] D'EON E., IRviNG G.: A quantized-diffusion model for rendering translucent materials. ACM Transactions on Graphics 30, 4 (2011), 56:1-56:14.

[DJ05] Donner C., Jensen H. W.: Light diffusion in multilayered translucent materials. ACM Transactions on Graphics 24, 3 (July 2005), 1032-1039.

[DJ07] Donner C., Jensen H. W.: Rendering translucent materials using photon diffusion. In Proceedings of Eurographics Symposium on Rendering (2007), pp. 243-251.

[dLE07] D'Eon E., LuebKe D., ENDERTon E.: Efficient rendering of human skin. In Proceedings of Eurographics Symposium on Rendering (2007), pp. 147-157.

[DLR*09] Donner C., Lawrence J., Ramamoorthi R., Hachisuka T., Jensen H. W., NAYAR S.: An empirical BSSRDF model. ACM Transactions on Graphics 28, 3 (July 2009), 30:1-30:10.

[DWd*08] Donner C., Weyrich T., D'Eon E., RamamoorTHI R., RUSINKIEWICZ S.: A layered, heterogeneous reflectance model for acquiring and rendering human skin. ACM Transactions on Graphics 27, 5 (2008), 140:1-140:12.

[GHP*08] Ghosh A., Hawkins T., Peers P., Frederiksen S., DEBEVEC P.: Practical modeling and acquisition of layered facial reflectance. ACM Transactions on Graphics 27, 5 (Dec. 2008), 139:1-139:10.

[GKD07] Green P., Kautz J., Durand F.: Efficient reflectance and visibility approximations for environment map rendering. Computer Graphics Forum 26, 3 (2007), 495-502.

[HK93] Hanrahan P., KRUEger W.: Reflection from layered surfaces due to subsurface scattering. In Proceedings of SIGGRAPH (1993), pp. 165-174. 
[JAM*10] JAKOB W., ARBREE A., MOON J. T., BALA K., MARSCHNER S.: A radiative transfer framework for rendering materials with anisotropic structure. ACM Trans. Graph. 29 (2010), 53:1-53:13

[JB02] Jensen H. W., BUHLER J.: A rapid hierarchical rendering technique for translucent materials. ACM Transactions on Graphics 21, 3 (2002), 576-581.

[JMLH01] JENSEN H. W., MARSChNER S. R., LEVOY M., HANRAHAN P.: A practical model for subsurface light transport. In Proceedings of SIGGRAPH 2001 (August 2001), pp. 511518

[JSB*10] Jimenez J., SCully T., Barbosa N., Donner C., Alvarez X., Vieira T., Matts P., Orvalho V., GutierREZ D., WEYRICH T.: A practical appearance model for dynamic facial color. ACM Transactions on Graphics 29, 6 (Dec. 2010), 141:1-141:10.

[LPT05] Li H., Pellacini F., Torrance K. E.: A hybrid Monte Carlo method for accurate and efficient subsurface scattering. In Rendering Techniques (June 2005), pp. 283-290.

[LSR*12] Li D., Sun X., REN Z., Lin S., TONG Y., Guo B., ZHOU K.: Transcut: Interactive rendering of translucent cutouts. IEEE Transactions on Visualization and Computer Graphics 99 , PrePrints (2012).

[PH00] PharR M., Hanrahan P. M.: Monte Carlo evaluation of non-linear scattering equations for subsurface reflection. In Proceedings of SIGGRAPH (2000), pp. 275-286.

[PvBM*06] Peers P., vom Berge K., Matusik W., RaMAMOORTHI R., LAWRENCE J., RUSINKIEWICZ S., DUTRÉ P.: A compact factored representation of heterogeneous subsurface scattering. ACM Transactions on Graphics 25, 3 (July 2006), 746-753.

[RZL*10] REN Z., ZHOU K., LI T., HUA W., GUO B.: Interactive hair rendering under environment lighting. In $A C M S I G-$ GRAPH 2010 papers (New York, NY, USA, 2010), SIGGRAPH '10, ACM, pp. 55:1-55:8

[Sta95] StAM J.: Multiple scattering as a diffusion process. In Proceedings of Eurographics Workshop on Rendering (1995), Eurographics, pp. 41-50.

[STPP09] Song Y., Tong X., Pellacini F., Peers P.: Subedit: a representation for editing measured heterogeneous subsurface scattering. ACM Transactions on Graphics 28, 3 (2009), 31:1-31:10.

[SZLG10] Sun X., Zhou K., Lin S., Guo B.: Line space gathering for single scattering in large scenes. In $A C M$ SIGGRAPH 2010 papers (New York, NY, USA, 2010), SIGGRAPH '10, ACM, pp. 54:1-54:8.

[TS06] TSAI Y.-T., SHIH Z.-C.: All-frequency precomputed radiance transfer using spherical radial basis functions and clustered tensor approximation. ACM Trans. Graph. 25, 3 (2006), 967-976.

[TWL*05] TONG X., WANG J., Lin S., Guo B., Shum H.-Y.: Modeling and rendering of quasi-homogeneous materials. ACM Transactions on Graphics 24, 3 (July 2005), 1054-1061.

[WCPW*08] Wang R., Cheslack-Postava E., Wang R., Luebke D., Chen Q., Hua W., Peng Q., BaO H.: Real-time editing and relighting of homogeneous translucent materials. the Visual Computer 24, 7 (July 2008), 565-575.

[WRG*09] Wang J., Ren P., Gong M., Snyder J., Guo B.: All-frequency rendering of dynamic, spatially-varying reflectance. ACM Trans. Graph. 28, 5 (2009), 133:1-133:10.
[WTL05] WANG R., TRAN J., LUEB KE D.: All-frequency interactive relighting of translucent objects with single and multiple scattering. ACM Transactions on Graphics 24, 3 (July 2005), 1202-1207.

[WWH*10] Wang Y., Wang J., Holzschuch N., Subr K., YONG J.-H., GUO B.: Real-time rendering of heterogeneous translucent objects with arbitrary shapes. Computer Graphics Forum 29, 2 (2010), 497-506.

[WZT*08] WANG J., ZhaO S., TONG X., Lin S., Lin Z., Dong Y., GuO B., Shum H.-Y.: Modeling and rendering of heterogeneous translucent materials using the diffusion equation. $A C M$ Transactions on Graphics 27, 1 (2008), 9:1-9:18.

[XGL*07] XU K., GaO Y., Li Y., JU T., HU S.-M.: Real-time homogenous translucent material editing. Computer Graphics Forum 26, 3 (2007), 545-552

[XJF*08] XU K., JIA Y., FU H., HU S., TAI C.: Spherical piecewise constant basis functions for all-frequency precomputed radiance transfer. IEEE Transactions on Visualization and Computer Graphics 14, 2 (2008), 454-467.

[XMR*11] Xu K., Ma L.-Q., Ren B., WAng R., Hu S.-M.: Interactive hair rendering and appearance editing under environment lighting. ACM Transactions on Graphics 30, 6 (2011), 173:1-173:10.

\section{Appendix}

A. Integral of an SG. The integral of an SG over the entire unit sphere is given by:

$$
\int_{\Omega} G\left(\mathbf{i} ; \mathbf{i}_{1}, \lambda\right) \mathrm{d} \mathbf{i}=\frac{2 \pi}{\lambda}\left(1-e^{-2 \lambda}\right)
$$

The integral of an SG multiplied by a cosine term over the entire unit sphere is given by:

$$
\int_{\Omega} G\left(\mathbf{i} ; \mathbf{i}_{1}, \lambda\right)\left(\mathbf{i} \cdot \mathbf{i}_{2}\right) \mathrm{d} \mathbf{i}=\frac{2 \pi\left(\mathbf{i}_{\mathbf{1}} \cdot \mathbf{i}_{2}\right)}{\lambda^{2}}\left(\lambda-1+(\lambda+1) e^{-2 \lambda}\right)
$$

B. Product of two SGs. The product of two SGs is still an $\mathrm{SG}$, given by $G\left(\mathbf{i} ; \mathbf{i}_{1}, \lambda_{1}\right) G\left(\mathbf{i} ; \mathbf{i}_{2}, \lambda_{2}\right)=c_{3} G\left(\mathbf{i} ; \mathbf{i}_{3}, \lambda_{3}\right)$, where:

$$
\begin{array}{r}
c_{3}=e^{\lambda_{3}-\left(\lambda_{1}+\lambda_{2}\right)}, \mathbf{i}_{3}=\frac{\lambda_{1} \mathbf{i}_{1}+\lambda_{2} \mathbf{i}_{2}}{\lambda_{3}}, \\
\lambda_{3}=\sqrt{\lambda_{1}^{2}+\lambda_{2}^{2}+2 \lambda_{1} \lambda_{2}\left(\mathbf{i}_{1} \cdot \mathbf{i}_{2}\right)}
\end{array}
$$

C. Product integral of two SGs. The product integral of two SGs is given by:

$$
\int_{\Omega} G\left(\mathbf{i} ; \mathbf{i}_{1}, \lambda_{1}\right) \cdot G\left(\mathbf{i} ; \mathbf{i}_{2}, \lambda_{2}\right) \mathrm{d} \mathbf{i}=\int_{\Omega} c_{3} G\left(\mathbf{i}, \mathbf{i}_{3}, \lambda_{3}\right) \mathrm{d} \mathbf{i}=\frac{2 \pi c_{3}}{\lambda_{3}}\left(1-e^{-2 \lambda_{3}}\right)
$$

where $c_{3}$ and $\lambda_{3}$ are the same as in $\mathbf{B}$ above. The product integral of two SGs weighted by a cosine term can be approximated by:

$$
\begin{aligned}
& \int_{\Omega} G\left(\mathbf{i} ; \mathbf{i}_{1}, \lambda_{1}\right) \cdot G\left(\mathbf{i} ; \mathbf{i}_{2}, \lambda_{2}\right) \cdot\left(\mathbf{i} \cdot \mathbf{i}_{4}\right) \mathrm{d} \mathbf{i}=\int_{\Omega} c_{3} G\left(\mathbf{i} ; \mathbf{i}_{3}, \lambda_{3}\right) \cdot\left(\mathbf{i} \cdot \mathbf{i}_{4}\right) \mathrm{d} \mathbf{i} \\
& \quad \approx\left(\mathbf{i}_{3} \cdot \mathbf{i}_{4}\right) \int_{\Omega} c_{3} G\left(\mathbf{i} ; \mathbf{i}_{3}, \lambda_{3}\right) \mathrm{d} \mathbf{i} \approx \frac{2 \pi c_{3}\left(\mathbf{i}_{3} \cdot \mathbf{i}_{4}\right)}{\lambda_{3}}\left(1-e^{-2 \lambda_{3}}\right) \\
& \approx 2 \pi\left(\lambda_{1}\left(\mathbf{i}_{1} \cdot \mathbf{i}_{4}\right)+\lambda_{2}\left(\mathbf{i}_{2} \cdot \mathbf{i}_{4}\right)\right) \cdot \frac{e^{\lambda_{3}-\left(\lambda_{1}+\lambda_{2}\right)}}{\lambda_{3}^{2}}\left(1-e^{-2 \lambda_{3}}\right)
\end{aligned}
$$


In this equation, the cosine function $\mathbf{i} \cdot \mathbf{i}_{\mathbf{4}}$ is usually much smoother than the SG, so we can directly approximate it using a constant value at the SG center (e.g. $\left.\mathbf{i}_{3} \cdot \mathbf{i}_{4}\right)$, and then it can be pulled out of the integral.

D. Integral of 1D Gaussian. The indefinite integral of a 1D Gaussian $g(x ; u, \lambda)=\exp \left(-\lambda(x-u)^{2}\right)$ is:

$$
\int g(x ; u, \lambda) \mathrm{d} x=\frac{1}{2} \sqrt{\frac{\pi}{\lambda}} \operatorname{erf}(\sqrt{\lambda}(x-u))
$$

where erf is the error function defined as: $\operatorname{erf}(x)=$ $\frac{2}{\sqrt{\pi}} \int_{0}^{x} e^{-t^{2}} \mathrm{~d} t$. The indefinite product integral of a 1D Gaussian with a linear function is defined as:

$\int x \cdot g(x ; u, \lambda) \mathrm{d} x=-\frac{1}{2 \lambda} g(x ; u, \lambda)+\frac{1}{2} \sqrt{\frac{\pi}{\lambda}} \operatorname{erf}(\sqrt{\lambda}(x-u))$

E. Refracted SG. According to Snell's law, the refracted direction $\mathbf{i}^{\prime}$ can be written as a function of the incident direction i:

$$
\mathbf{i}^{\prime}=\psi(\mathbf{i})=\eta \mathbf{i}+\left(\cos \theta_{2}-\eta \cos \theta_{1}\right) \mathbf{n}
$$

where $\eta$ is the relative index of refraction; $\theta_{1}$ and $\theta_{2}$ are the incident and refracted angles respectively. They are defined as:

$$
\cos \theta_{1}=\mathbf{i} \cdot \mathbf{n}, \quad \cos \theta_{2}=\sqrt{1-\eta^{2}\left(1-\cos ^{2} \theta_{1}\right)}
$$

When expressing the incident lighting function as an SG: $G\left(\mathbf{i} ; \mathbf{i}_{\mathbf{j}}, \lambda_{j}\right)$, the refracted lighting function can be modeled as a warped lobe of the incident SG and approximated by an SG itself:

$$
G\left(\psi^{-1}\left(\mathbf{i}^{\prime}\right) ; \mathbf{i}_{\mathbf{j}}, \lambda_{j}\right) \approx G\left(\mathbf{i}^{\prime} ; \mathbf{i}_{\mathbf{j}}^{\prime}, \lambda_{j}^{\prime}\right)
$$

In this approximation, we preserve both the center and amplitude of the warped lobe. The bandwidth parameter is determined by preserving local curvature, which is:

$$
\mathbf{i}_{\mathbf{j}}^{\prime}=\psi\left(\mathbf{i}_{\mathbf{j}}\right), \quad \lambda_{j}^{\prime}=\lambda_{j} \cdot\left(\left.\frac{\mathrm{di} \mathbf{i}^{\prime}}{\mathrm{di}}\right|_{\mathbf{i}=\mathbf{i}_{\mathbf{j}}}\right)=\frac{\lambda_{j} \eta^{2} \cos \theta_{1}}{\cos \theta_{2}}
$$

